\title{
AdS/CFT correspondence in operator formalism
}

\section{Seiji Terashima}

Yukawa Institute for Theoretical Physics, Kyoto University, Kyoto 606-8502, Japan

E-mail: terasima@yukawa.kyoto-u.ac.jp

ABSTRACT: In this paper we study the AdS/CFT correspondence in the operator formalism without assuming the GKPW relation. We explicitly show that the low energy spectrum of the large $N$ limit of CFT, which is realized by a strong coupling gauge theory, is identical to the spectrum of the free gravitational theory in the global AdS spacetime under some assumptions which are expected to be valid. Thus, two theories are equivalent for the low energy region under the assumptions. Using this equivalence, the bulk local field is constructed and the GKPW relation is derived.

KeYwords: AdS-CFT Correspondence, 1/N Expansion, Gauge-gravity correspondence

ArXiv EPrint: 1710.07298 


\section{Contents}

1 Introduction and summary 1

2 Free fields on $\mathbf{A d S}_{d+1} \quad 3$

2.1 Scalar field 4

2.2 Gauge field 5

2.3 Gravitational perturbation 6

$\begin{array}{llr}3 & \text { Spectrum of } \mathbf{C F T}_{d} & \mathbf{7}\end{array}$

3.1 Construction of bulk local field 11

3.2 Derivation of GKPW relation 13

$\begin{array}{lll}3.3 \text { Current } & 15\end{array}$

$\begin{array}{lll}3.4 & \text { Energy-momentum tensor } & 15\end{array}$

$\begin{array}{lll}4 & \mathrm{AdS}_{3} / \mathrm{CFT}_{2} & 17\end{array}$

$\begin{array}{llr}5 & \text { Thermal states } & 18\end{array}$

$\begin{array}{ll}\text { A Symmetric tensor harmonics on } S^{d-1} & 19\end{array}$

$\begin{array}{ll}\text { B Normalization of spherical harmonics } & 19\end{array}$

\section{Introduction and summary}

The AdS/CFT correspondence is the conjecture which claims the equivalence between a $d$ dimensional conformal field theory $\left(\mathrm{CFT}_{d}\right)$ and a $d+1$-dimensional quantum gravity on an asymptotically $\mathrm{AdS}_{d+1}$ spacetime [1]. This surprising conjecture is highly non-trivial and important in various aspects of physics. In particular, according to this correspondence, we have concrete examples of quantum gravities in terms of $\mathrm{CFT}_{d}$ which is much better understood. Thus, this conjecture has been investigated intensively and there are many evidences for this conjecture, although there is no proof.

In the most popular formulation of the AdS/CFT correspondence is the GKPW relation $[2,3]$ where the CFT partition function with the source terms is identified with the quantum gravity partition function on AdS with appropriate boundary conditions corresponding to the source terms. This is in particular useful for Euclidean $\mathrm{AdS}_{d+1}$ space. Another formulation of the AdS/CFT correspondence is the equivalence between the Hilbert spaces and the Hamiltonians of the two theories in the operator formalism. For this, we need to choose a time direction and the usual choice is the $\mathrm{CFT}_{d}$ on $\mathbf{R} \times S^{d-1}$ where $\mathbf{R}$ 
represents the time. ${ }^{1}$ This was initiated in $[4,5]$. In particular, in [5] it was stated that the energy spectrum of the $\mathrm{CFT}_{d}$ which satisfies the large $N$ factorization is identified with the spectrum on free theories in $\mathrm{AdS}_{d+1}$ space because both of them should be representations of the conformal symmetry. ${ }^{2}$

In this paper, we study the AdS/CFT correspondence for the weak gravity limit in the global $\mathrm{AdS}_{d+1}$ space in the operator formalism without assuming the GKPW relations. ${ }^{3}$ First, we explicitly show that the (low energy) spectrum of the large $N \mathrm{CFT}_{d}$ is identical to the spectrum of the free gravitational theory in global $\mathrm{AdS}_{d+1}$ under three assumptions which are expected to be valid for the large $N$ gauge theory. Thus, two theories are equivalent at least for the low energy region if we accept the assumptions. Here, the first assumption is that the low energy spectrum is determined only by the conserved symmetry currents whose conformal dimension is protected against the quantum corrections. ${ }^{4}$ The second one is the large $N$ factorization of the correlators. The last one is that the spectrum generated from the primary states by acting the conformal symmetry generators is completely independent except the relations given by the symmetry. These assumptions are very natural for the large $N$ strongly coupled gauge theories. ${ }^{5}$

With this explicit identification of the spectrum of CFT to the spectrum of the AdS space, we can construct the localized state of the AdS space as a state of the CFT. This construction of the localized state coincides with the known results [8-10]. Furthermore, using the identification, we can construct the local field in AdS space from the operators in CFT. The bulk reconstruction of the local field has been intensively studied, for example, in [11]-[23] where a version of the GKPW relation, which implies that the boundary value of the bulk field is the CFT primary field, [5] was used. ${ }^{6}$ In this paper, we start from the identification of the spectrum, therefore, the GKPW is not assumed. Instead, we will show that the GKPW relation is a consequence of the above three assumptions.

For the $\mathrm{AdS}_{3} / \mathrm{CFT}_{2}$ case, our general consideration is not applicable to both the gravitons in $\mathrm{AdS}_{3}$ and the energy-momentum tensor in $\mathrm{CFT}_{2}$. We also show that the equivalence of the two theories under the above assumptions.

For the background other than the vacuum, it is not straightforward to extend this identification of the states and the Hamiltonian because we can not explicitly construct states in CFT corresponding to excitations around the black hole background. Although this difficulty, we give some interpretation of a qualitative counting of the states around

\footnotetext{
${ }^{1}$ If we are interested in the states and the Hamiltonian of the theory, the Euclidean and Lorentzian theories need not to be distinguished because these two theories have same states and the Hamiltonian, but the time translation operators are different by the factor $\sqrt{-1}$.

${ }^{2}$ There are many important papers which study the derivation of the theory on AdS from CFT, in particular for including interactions, for example, in $[6,7]$.

${ }^{3}$ In this paper, we will focus on the leading order in the large $N$ limit only for simplicity.

${ }^{4}$ Only the symmetry currents considered in this paper are usual spin one currents and the energymomentum tensor, just for the simplicity. We hope that other symmetries will be investigated in near future.

${ }^{5}$ For $\mathrm{CFT}_{d}$ which is not given by a gauge theory, first two assumptions can be replaced by the sparseness condition of the spectrum and the requirement that the theory is the generalized free theory.

${ }^{6}$ Technically, our computations in this paper would be regarded as an inverse of the computations of HKLL [13].
} 
the typical states which represent a thermal state in CFT and corresponding black hole background.

This paper is organized as follows. In the next section we reviewed the spectrum of the free theories on the global $\mathrm{AdS}_{d+1}$ spacetime. In section three, we consider the spectrum of the $\mathrm{CFT}_{d}$ which is realized by a $d$-dimensional large $N$ strong coupling gauge theory on $S^{d-1}$ space. Under the natural assumptions, this spectrum is identified with the bulk spectrum. Using this identification, the bulk local field is constructed and the GKPW relation is derived. For the $\mathrm{AdS}_{3}$ case, we need special care as discussed in section four. Extension of the analysis to the thermal states in $\mathrm{CFT}_{d}$ is discussed in the final section. In the appendices, some properties of the symmetric tensor harmonics are reviewed.

\section{Free fields on $\mathrm{AdS}_{d+1}$}

In this section, we will give the spectrum of the free theory limit of the gravitational theory on the global $\mathrm{AdS}_{d+1}$. In the next section, we will see that a spectrum of a large $N \mathrm{CFT}_{d}$ is same as this spectrum if we assume some properties of the theory. This computations of spectrum of the theory on the global $\mathrm{AdS}_{d+1}$ have been done for scalar theory in $[4,24]$. For the gauge field and the metric, we will mainly use the explicit results given in [25] in which they used the gauge invariant formalism. We will show their results in a way such that the comparison with the spectrum of $\mathrm{CFT}_{d}$ is easier. The action of the gravitational theory is

$$
S_{\text {grav }}=\frac{1}{16 \pi G_{N}} \int d^{d+1} x \sqrt{-\operatorname{det} g}(R+2 \Lambda)
$$

where $\Lambda=-\frac{d(d+1)}{2 l_{\mathrm{AdS}}^{2}}$ and we set the AdS scale $l_{\mathrm{AdS}}=1$ in this paper. The metric of the vacuum solution is the $\mathrm{AdS}_{d+1}$ metric:

$$
d s_{\mathrm{AdS}}^{2}=-\left(1+r^{2}\right) d t^{2}+\frac{1}{1+r^{2}} d r^{2}+r^{2} d \Omega_{d-1}^{2},
$$

where $0 \leq r<\infty,-\infty<t<\infty$ and $d \Omega_{d-1}^{2}$ is the metric for the $d$-1-dimensional round unit sphere $S^{d-1}$ By the coordinate change $r=\tan \rho$, the metric is also written as

$$
d s_{\mathrm{AdS}}^{2}=\frac{1}{\cos ^{2}(\rho)}\left(-d t^{2}+d \rho^{2}+\sin ^{2}(\rho) d \Omega_{d-1}^{2}\right),
$$

where $0 \leq \rho<\pi / 2 .{ }^{7}$ The boundary of the $\operatorname{AdS}_{d+1}$ is located at $\rho=\pi / 2$.

We will also consider the scalar and the gauge field which coupled the gravity. In this paper, we take the limit where the gravitational coupling vanishes, i.e. $G_{N} \rightarrow 0$, around this background and find the spectrum of the corresponding free theory. (All non-normalized modes of the fields are assumed to vanish.) Here, other coupling constants in the theory are also taken to vanish.

\footnotetext{
${ }^{7}$ The coordinate $x$ taken in [25] is $x=\pi / 2-\rho$. Note that they defined $D-d+1$ and $n=d-1$ in [25].
} 


\subsection{Scalar field}

The action of the free scalar field is given by

$$
S_{\text {scalar }}=\int d^{d+1} x \sqrt{-\operatorname{det}(g)}\left(\frac{1}{2} g^{M N} \nabla_{M} \phi \nabla_{N} \phi+\frac{m^{2}}{2} \phi^{2}\right),
$$

where $M, N=1, \cdots, d+1$ and the e.o.m. is

$$
0=-g^{M N} \nabla_{M} \nabla_{N} \phi+m^{2} \phi^{2}
$$

We expand $\phi$ with the spherical harmonics $Y_{l m}(\Omega)$,

$$
\phi(t, \rho, \Omega)=\sum_{n, l, m}\left(a_{n l m}^{\dagger} e^{i \omega_{n l} t}+a_{n l m} e^{-i \omega_{n l} t}\right) \psi_{n l m}(\rho) Y_{l m}(\Omega),
$$

where $\Omega$ represents the coordinates of $S^{d-1}$. Then, rewriting the radial functions as

$$
\psi_{n l m}(\rho)=(\tan (\rho))^{-\frac{d-1}{2}} \Phi_{n l m}(\rho),
$$

the e.o.m. is reduced to

$$
\begin{aligned}
\left(\omega_{n l}^{2}+\frac{\partial^{2}}{\partial \rho^{2}}-\frac{1}{\sin ^{2} \rho}(l(l+d\right. & \left.-2)+\frac{(d-1)(d-3)}{4}\right) \\
& \left.-\frac{1}{\cos ^{2} \rho}\left(m^{2}+\frac{d^{2}-1}{4}\right)\right) \Phi_{n l m}(\rho)=0
\end{aligned}
$$

where $\omega_{n l} \geq 0$ and $n, l=0,1,2, \ldots$. As we will see later, this equation appears for the e.o.m. of the gauge fields and gravitons. The normalized solution for the e.o.m. is given with the Gauss's hyper geometric function as

$$
\psi_{n l m}(\rho)=\frac{1}{N_{n l}} \sin ^{l}(\rho) \cos ^{\Delta}(\rho){ }_{2} F_{1}\left(-n, \Delta+l+n, l+\frac{d}{2}, \sin ^{2}(\rho)\right),
$$

where $\Delta$ is given by the equation

$$
m^{2}=\Delta(\Delta-d)
$$

i.e. $\Delta=d / 2 \pm \sqrt{m^{2}+d^{2} / 4}$, and

$$
\omega_{n l}=\Delta+l+2 n
$$

Here, we assume the Breitenlohner-Freedman bound [24] $m^{2}+d^{2} / 4 \geq 0$ is satisfied. If $m^{2}+d^{2} / 4 \geq 1$ is satisfied, the solution correspond $\Delta=d / 2-\sqrt{m^{2}+d^{2} / 4}(<d / 2-1)$ is non-normalizable. We will consider this case only in this paper for simplicity and take $\Delta=d / 2+\sqrt{m^{2}+d^{2} / 4} \cdot{ }^{8}$ Using the standard inner product,

$$
\left(u_{1}, u_{2}\right)=i \int_{\Sigma} \sqrt{-\operatorname{det} g} g^{t t}\left(u_{1}^{*} D_{t} u_{2}-D_{t} u_{1}^{*} u_{2}\right),
$$

\footnotetext{
${ }^{8}$ As we will see later that the relevant operator in the $\mathrm{CFT}_{d}$ corresponds to $\Delta<d$, which implies $m^{2}<0$. the irrelevant operator corresponds to $m^{2}>0$.
} 
where $\Sigma$ is a space-like slice in $\mathrm{AdS}_{d+1}$, the normalization constant is given by

$$
N_{n l}=(-1)^{n} \sqrt{\frac{n ! \Gamma\left(l+\frac{d}{2}\right)^{2} \Gamma\left(\Delta+n+1-\frac{d}{2}\right)}{\Gamma\left(n+l+\frac{d}{2}\right) \Gamma(\Delta+n+l)}},
$$

where we have chosen the phase of $N_{n l}$, which can be any value, as in [18]. Then, in the $G_{N} \rightarrow 0$ limit we have the quantized free scalar field

$$
\hat{\phi}(t, \rho, \Omega)=\sum_{n, l, m}\left(\hat{a}_{n l m}^{\dagger} e^{i \omega_{n} t}+\hat{a}_{n l m} e^{-i \omega_{n} t}\right) \psi_{n l m}(\rho) Y_{l m}(\Omega),
$$

with the commutation relation

$$
\left[\hat{a}_{n l m}, \hat{a}_{n^{\prime} l^{\prime} m^{\prime}}^{\dagger}\right]=\delta_{n, n^{\prime}} \delta_{l, l^{\prime}} \delta_{m, m^{\prime}}
$$

and the Hamiltonian such that

$$
\left[\hat{H}, \hat{a}_{n l m}\right]=-\omega_{n l} .
$$

The Hilbert space is the Fock space spanned by $\prod_{n, l, m}\left(\hat{a}_{n l m}^{\dagger}\right)^{\mathcal{N}_{n l m}}|0\rangle$, where $\mathcal{N}_{n l m}$ is a nonnegative integer. We choose the constant shift of the Hamiltonian as $\hat{H}|0\rangle=0$ where $|0\rangle$ is the vacuum, i.e. $\hat{a}_{n l m}|0\rangle=0$.

\subsection{Gauge field}

The action of the free abelian gauge field is given by

$$
S=\int d^{d+1} x \sqrt{-\operatorname{det}(g)}\left(\frac{1}{2} g^{M N} g^{M^{\prime} N^{\prime}} F_{M M^{\prime}} F_{N N^{\prime}}\right),
$$

and the e.o.m. is the Maxwell equation:

$$
0=\nabla_{M} F^{M N}
$$

where

$$
F_{M N}=\nabla_{M} A_{N}-\nabla_{N} A_{M}
$$

Note that the non-abelian gauge field becomes a collection of the abelian gauge fields in the free limit.

We will follow [25] for the analysis of the perturbation around $A_{M}=0$ for $d \geq 2$. In [25], the coordinates with the following metric were used:

$$
d s_{\mathrm{AdS}}^{2}=g_{a b} d y^{a} d y^{b}+r^{2} g_{i j} d z^{i} d z^{j},
$$

where the indices $a, b(=1,2)$ are for the $\mathrm{AdS}_{2}$ part and $z^{i}$ is the coordinates for $S^{d-1}$. The 1-form gauge field $A$ is decomposed into the representations of $\mathrm{SO}(d)$ action on $S^{d-1}$ as

$$
A=A_{M} d x^{M}=A_{M}^{V} d x^{M}+A_{M}^{S} d x^{M},
$$


where

$$
\begin{aligned}
& A_{M}^{V} d x^{M}=\sum_{l, m} \phi_{l m}^{V}(y) Y_{i}^{l m}(z) d z^{i}, \\
& A_{M}^{S} d x^{M}=\sum_{l, m} A_{l m a}^{S}(y) Y^{l m}(z) d y^{a}+\sum_{l, m} A_{l m}^{S}(y) D_{i} Y^{l m}(z) d z^{i} .
\end{aligned}
$$

In this expression, we used the coordinates $(2.20)$ and $D_{i}$ is the covariant derivative on $S^{d-1}$. The transverse vector spherical harmonic $Y_{i}^{l m}$ is defined in appendix A and the index $m$ represents the label of the spherical harmonics with the "angular momentum" $l$.

The gauge transformation, $A \rightarrow A+d \lambda$, only affects the $A_{\mu}^{S}$ part. In [25], the gauge invariant for $A^{S}$ and $A_{a}^{S}$ is shown to be $\phi^{S}$ (up to a constant shift) which satisfies

$$
D_{a} \phi_{l m}^{S}=\epsilon_{a b} r^{d-3}\left(D^{b} A_{l m}^{S}+A_{l m}^{S b}\right),
$$

where $\epsilon_{a b}$ is the metric compatible volume element on the $\mathrm{AdS}_{2}$ part with the metric $g_{a b}$. Then, the equations of motion for $\Phi_{l m}^{V}=r^{(d-3) / 2} \phi_{l m}^{V}$ is reduced to the equation (2.8) for the scalar field with $m^{2}=1-d$. For $\Phi_{l m}^{S}=r^{-(d-3) / 2} \phi_{l m}^{S}$, it is reduced to (2.8) with $m^{2}=-2(d-2)$. Thus, the mode of $\Phi^{S}$ for $\Delta=d / 2-\sqrt{m^{2}+d^{2} / 4}$ is non-normalizable and then we should choose $\Delta=d / 2+\sqrt{m^{2}+d^{2} / 4}$. With this choice, we obtain $\Delta=d-1$ and $\Delta=d-2$ for $\Phi^{V}$ and $\Phi^{S}$, respectively.

Note that this analysis is not valid for the scalar mode with $l=0$. This is because $Y^{l m}$ is a constant, then $D_{i} Y^{l m}=0$ for this mode. This means that this mode is a two dimensional gauge field $A^{S} \sim A_{a}^{S}(y) d y^{a}$, which has no fluctuating modes because we fixed the boundary condition for the gauge field.

Therefore, the spectrum of the one particle states is given by

$$
\omega_{n l}=d-1+l+2 n,
$$

for $\Phi^{V}$, which is the representation of $\mathrm{SO}(d)$ corresponding to the Young diagram labeled by $[l, 1,0, \ldots, 0]$, and

$$
\omega_{n l}=d-2+l+2 n
$$

for $\Phi^{S}$, which corresponds to $[l, 0,0, \ldots, 0]$, where $l=1,2, \cdots$ and $n=0,1, \cdots$ for both of $\Phi^{V}$ and $\Phi^{S}$.

\subsection{Gravitational perturbation}

As for the gauge field, the fluctuations of the metric, $d s^{2}=g_{\mu \nu} d x^{\mu} d x^{\nu}+h_{\mu \nu} d x^{\mu} d x^{\nu}$, will be decomposed by the tensor harmonics on $S^{d-1} .{ }^{9}$ The explicit gauge invariant parametrization of the fluctuations are also constructed and the equations of motion for them were given in [25]. The fluctuations reduces to one tensor, one vector and one scalar modes on $S^{d-1}$. The e.o.m. for them become the equation (2.8) for the scalar field with $m^{2}=0,1-d$ and $-2(d-2)$ for the tensor, the vector and the scalar type perturbations, respectively.

\footnotetext{
${ }^{9}$ The analysis below is valid for $d \geq 3$.
} 
Here, $\Delta=d / 2+\sqrt{m^{2}+d^{2} / 4}$ should be chosen because the other choice corresponds to the non-normalizable mode. Furthermore, in [25] it was shown that there are no dynamical degrees of freedom for the scalar modes with $l=0,1$ and the vector modes with $l=1$.

As a result, the spectrum of the one particle states is

$$
\omega_{n l}=d+l+2 n,
$$

for the tensor type perturbation, which is the representation of $\mathrm{SO}(d)$ corresponding to the Young diagram labeled by $[l, 2,0, \ldots, 0]$,

$$
\omega_{n l}=d-1+l+2 n,
$$

for the vector type perturbation, which corresponds to $[l, 1,0, \ldots, 0]$, and

$$
\omega_{n l}=d-2+l+2 n,
$$

for the scalar type perturbation, which corresponds to $[l, 0,0, \ldots, 0]$, where $l=2,3, \cdots$ and $n=0,1, \cdots$ for all the three kinds of the perturbations.

\section{Spectrum of $\mathrm{CFT}_{d}$}

In this section, we will show the equivalence between the spectra of the free gravitational theory on $\mathrm{AdS}_{d+1}$ given in the previous section and the spectra of the $\mathrm{CFT}_{d}$. Using this equivalence, we will construct the local operators and local states in $\mathrm{AdS}_{d+1}$ in $\mathrm{CFT}_{d}$ point of view. It will also shown that the GKPW relation is derived from this equivalence.

Now, let us consider a $d$-dimensional $\mathrm{SU}(N)$ gauge theory with a conformal symmetry on $\mathbf{R} \times S^{d-1}$ where $\mathbf{R}$ is the time direction. Here the radius of $S^{d-1}$ is taken to be one. The matter contents and interactions of them are not specified and the gauge group can be another gauge group, say, $\mathrm{SO}(\mathrm{N}), \mathrm{USP}(2 \mathrm{~N})$ and a product of them. However, we will assume some conditions for the gauge theory later. For a review of the $\mathrm{CFT}_{d}$, see for example, [26-28]. We will use the (almost same) convention taken in [28], in particular, $P_{\mu}$ corresponds to $\partial_{\mu}$. The generators of the conformal symmetry are $\hat{D}, \hat{M}_{\mu \nu}, \hat{P}_{\mu}, \hat{K}_{\mu}$ which satisfy the following commutation relation

$$
\left[\hat{D}, \hat{P}_{\mu}\right]=\hat{P}_{\mu}, \quad\left[\hat{D}, \hat{K}_{\mu}\right]=-\hat{K}_{\mu}, \quad\left[\hat{K}_{\mu}, \hat{P}_{\nu}\right]=2 \delta_{\mu \nu} \hat{D}-2 \hat{M}_{\mu \nu}
$$

and the usual commutation relations for the $\mathrm{SO}(d)$ rotation with $\hat{M}_{\mu \nu}$. Note that for these conformal symmetry generators, which act on the states on $S^{d-1}$, we use the notation for the theory on flat $\mathbf{R}^{d}$, thus the $\mu, \nu$ indices take $1,2, \ldots, d$ and there is no distinction between the upper and lower ones. The Hamiltonian of the theory is the dilatation operator $\hat{H}=\hat{D}$ and $M_{\mu \nu}$ are the generators of the isometries of $S^{d-1}$. The (conformal) primary state $|\Delta\rangle$ is the state satisfies $\hat{K}_{\mu}|\Delta\rangle=0$ and $\hat{D}|\Delta\rangle=\Delta|\Delta\rangle$. This is obtained from the corresponding primary field $\mathcal{O}_{\Delta}(x)$ as ${ }^{10}$

$$
\lim _{x \rightarrow 0} \mathcal{O}_{\Delta}(x)|0\rangle=|\Delta\rangle=\hat{\mathcal{O}}_{\Delta}|0\rangle,
$$

\footnotetext{
${ }^{10}$ Here the field is defined as the radial quantization on the flat Euclidean $\mathbf{R}^{d}$.
} 
where the conformal vacuum satisfies $\hat{K}^{\mu}|0\rangle=\hat{D}|0\rangle=\hat{M}^{\mu \nu}|0\rangle=0$. Here we defined the operator

$$
\hat{\mathcal{O}}_{\Delta}=\lim _{x \rightarrow 0} \hat{\mathcal{O}}_{\Delta}^{+}(x)
$$

where $\hat{\mathcal{O}}_{\Delta}^{+}(x)$ is the regular parts of $\mathcal{O}_{\Delta}(x)$ in $x^{\mu} \rightarrow 0$ limit which can be expanded by the polynomial of $x^{\mu}{ }^{11}$ It is also required that $\left(\mathcal{O}_{\Delta}(x)-\hat{\mathcal{O}}_{\Delta}^{+}(x)\right)|0\rangle=0$ for the regularity at $x=0$. This operator satisfies

$$
\hat{K}_{\mu} \hat{\mathcal{O}}_{\Delta}|0\rangle=0, \quad\left[\hat{H}, \hat{\mathcal{O}}_{\Delta}\right]=\Delta
$$

Note that $\left[\hat{K}_{\mu}, \hat{\mathcal{O}}_{\Delta}\right] \neq 0$, except for a large $N$ limit. We also note that composite operators of $\mathcal{O}_{\Delta}(x)$ can be primary fields. Any state in CFT can be obtained from a primary state by acting $\hat{P}^{\mu}$

$$
\hat{P}^{\left(\mu_{1}\right.} \hat{P}^{\mu_{2}} \ldots \hat{P}^{\left.\mu_{l}\right)}|\Delta\rangle
$$

where the parenthesis means the symmetrization of the indices because $\left[\hat{P}_{\mu}, \hat{P}_{\nu}\right]=0$.

Now let us consider the energy spectrum of the CFT in a large $N$ limit. In this limit, the energy (i.e. the eigenvalue of $\hat{D}$ ) of a generic state is expected to diverge because the quantum effects depend on $N$. Only the exceptions will be the symmetry protected states which has the energy of $\mathcal{O}\left(N^{0}\right) .{ }^{12}$ Because we consider a CFT, there is always energymomentum tensor $T_{\mu \nu}(x)$ which is traceless and the primary field with $\Delta=d$. If there is a global symmetry, the corresponding conserved current $J_{\mu}(x)$ is the primary field with $\Delta=d-1$. Other symmetries including supersymmetries and higher spin symmetries ensures the corresponding primary fields with finite conformal dimensions. Furthermore, the supersymmtric theory with many super charges may have BPS states whose dimensions are also protected. In this paper, we concentrate the current and the energy momentum tensor for simplicity, and assume that only the symmetry currents are the spectrum of order $\mathcal{O}\left(N^{0}\right) .{ }^{13}$ We also consider scalar fields in this paper. We will regard these are associated with the conserved current by supersymmetry or whose conformal dimensions are accidentally low.

Another assumption we impose is the large $N$ factorization. For a large $N$ gauge theory, the large $N$ factorization occurs, at least, in a perturbation theory or a semiclassical computation. This implies that, in the large $N$ limit, correlators of the single trace operators which are defined by composite operators with one trace are approximated by the two point correlators for all pairings of the operators. ${ }^{14}$ Note that the symmetry

\footnotetext{
${ }^{11}$ For the stress energy tensor in two dimensional theory, $\hat{\mathcal{O}}_{\Delta}$ is $L_{-2}$ or $\tilde{L}_{-2}$.

${ }^{12}$ If we tune the parameters of the CFT, there will be other finite energy (primary) states. In particular, the double scaling limit gives the spectrum of the string theory. However, we will not consider such cases in this paper.

${ }^{13}$ This assumptions can be replaced by the sparse spectrum.

${ }^{14} \mathrm{~A}$ theory with this property is called generalized free theory. For a $\mathrm{CFT}_{d}$ which is not defined by a gauge theory, this property will be assumed. For generalized free theories, (3.6) is satisfied. For the generalized free theory and AdS/CFT correspondence, see, for example, [29].
} 
protected operators include single trace operators of the gauge theory. We assume this large $N$ factorization. Then, we can easily see that, in the large $N$ limit, the commutator should be proportional to the identity operator:

$$
\left[\hat{\mathcal{O}}_{\Delta_{a}}(x), \hat{\mathcal{O}}_{\Delta_{b}}(y)\right]=f(x-y),
$$

where $f(x-y)$ is a c-number function because of the Wick theorem which guarantees the vanishing of the connected $n$-point functions for $n>2$. We will not explicitly write down $f(x-y)$, however, the commutators for the mode expanded operators can be computed from the two-point function,

$$
\left\langle 0\left|\mathcal{O}_{\Delta_{a}}(x) \mathcal{O}_{\Delta_{b}}(y)\right| 0\right\rangle=\delta_{a b} \frac{1}{(x-y)^{2 \Delta_{a}}},
$$

where the index $a, b$ labels the conserved currents in the $\mathrm{CFT}_{d}$. For example, we find

$$
\left[\hat{\mathcal{O}}_{\Delta_{a}},\left(\hat{\mathcal{O}}_{\Delta_{b}}\right)^{\dagger}\right]=\delta_{a b}
$$

We also find $\left[\hat{\mathcal{O}}_{\Delta_{a}}, \hat{\mathcal{O}}_{\Delta_{b}}\right]=0$.

Under the assumptions, the low energy, i.e. $\mathcal{O}\left(N^{0}\right)$, states in the large $N$ limit will be given by

$$
R\left(\hat{P}^{\mu}, \hat{\mathcal{O}}_{\Delta_{a}}\right)|0\rangle
$$

where $R$ is a polynomial. ${ }^{15}$ We expect that the states (3.9) are independent for the strongly interacting large $N$ CFT up to the commutation relations (3.6) and the symmetry relations, i.e. the conservation law and the traceless properties of the energy momentum tensor. This is because, for the large $N$ gauge theory, there will be no specific energy scale where a linear relation between the states appear. Note that the interaction is strong in the large $N$ limit with fixed coupling constants. We assume this complete independence of the states (except the symmetry relations) also.

It is clear that this complete independence is impossible for finite $N$ case because this implies that there are infinitely many primary fields. Furthermore, it is not a general property of a theory with infinitely many degrees of freedom which satisfies the large $N$ factorization (more generally, a generalized free theory). For example, for infinitely many free fields $\varphi_{a}$, the e.o.m. is $\hat{P}_{\mu} \hat{P}^{\mu} \hat{\varphi}_{a}|0\rangle=0$, and other similar relations hold. Then, the number of independent states is much smaller. On the other hand, the symmetry currents in a strongly interacting gauge theory will not satisfy any e.o.m. with a finite number of $\hat{P}^{\mu}$ in the large $N$ limit. Another example which does not satisfy the complete independence is the current algebra in $\mathrm{CFT}_{2}$. For this there are extra relations from the holomorphy. For example, there is a relation $\left[\tilde{L}_{-1}, J_{-1}\right]=0$, where $\tilde{L}_{-1}$ is a linear combination of $P^{1}$ and $P^{2}$ and $J_{n}$ is the holomorphic current.

Below, we will see that the spectrum of the $\mathrm{CFT}_{d}$ in the large $N$ limit is equivalent to the spectrum of a gravity theory on $\mathrm{AdS}_{d+1}$ in the free theory limit under these three assumptions.

\footnotetext{
${ }^{15}$ Of course, $\mathcal{O}_{\Delta_{b}}^{\dagger}$ does not appear because $\left[\hat{P}^{\mu}, \mathcal{O}_{\Delta_{b}}^{\dagger}\right]=0$ and $\mathcal{O}_{\Delta_{b}}^{\dagger}|0\rangle=0$.
} 
The identification of the CFT states to the states of the Fock space of the scalar fields in AdS is explicitly given by the identification of the raising operators as

$$
\hat{a}_{n l m}^{\dagger}=c_{n l} s_{(l, m)}^{\mu_{1} \mu_{2} \ldots \mu_{l}} P_{\mu_{1}} P_{\mu_{2}} \cdots P_{\mu_{l}}\left(P^{2}\right)^{n} \hat{\mathcal{O}}_{\Delta}
$$

where $c_{n l}$ is the normalization constant, which will be determined later, $P^{\mu}$ act on an operator such that $P^{\mu} \hat{\phi}=\left[\hat{P}^{\mu}, \hat{\phi}\right]$ and $s_{(l, m)}^{\mu_{1} \mu_{2} \ldots \mu_{l}}$ is a normalized rank $l$ symmetric traceless constant tensor. Indeed, the eigen value of the Hamiltonian is given by the correct one: ${ }^{16}$

$$
\left[\hat{H}, \hat{a}_{n l m}^{\dagger}\right]=\Delta+2 n+l .
$$

Note that this relation, i.e. $\hat{D}=\sum_{n, l, m} \hat{a}_{n l m}^{\dagger} \hat{a}_{n l m}$, is only valid for the modes with $\mathcal{O}\left(N^{0}\right)$ energy.

We can also see that $\hat{a}_{n l m}^{\dagger} \sim \hat{\mathcal{O}}_{\Delta n l m}$ where $\hat{\mathcal{O}}_{\Delta n l m}$ is defined by the coefficient of the expansion of $\hat{\mathcal{O}}_{\Delta}^{+}(x)$ around $x=0$ as follows $\hat{\mathcal{O}}_{\Delta}^{+}(x)=$ $\sum_{n, l, m} s_{(l, m)}^{\mu_{1} \mu_{2} \ldots \mu_{l}} x_{\mu_{1}} x_{\mu_{2}} \cdots x_{\mu_{l}}\left(x^{2}\right)^{n} \hat{\mathcal{O}}_{\Delta n l m}$. Furthermore, the states generated by $\mathcal{O}(x)$ are generated by $\hat{\mathcal{O}}_{\Delta n l m}$. Therefore, the Hilbert spaces of a fixed energy are identical for $\mathrm{CFT}_{d}$ and for the scalar in $\operatorname{AdS}_{d+1} \cdot{ }^{17}$

Note that $\left[\hat{a}^{\dagger}, \hat{a}\right]=1$ is also shown by appropriately fixing the normalization constant $c_{n l}$ because the commutators of the operators are proportional to the identity operator.

This equivalence is similar to the deconstruction of an extra dimension [30,31] or lattice field theories. ${ }^{18}$ For those theories, there are infinitely many fields, which are labeled by the nodes or the links, to construct the extra dimensions. Here, the only the one (or finitely many ) field $\mathcal{O}(x)$ corresponds to the field in the extra dimension. This seems strange. However, we assumed the complete independence in the large $N$ limit. Thus, the field $\mathcal{O}(x)$ does not satisfies any differential equation which will be regarded as an e.o.m. For example, the free theory has two modes, i.e. positive and negative frequency modes, for a fixed momentum given by the e.o.m. For the field $\mathcal{O}(x)$ in the large $N$ limit, there are indeed infinitely many modes for a fixed (angular) momentum, which are independent each other by the assumption. Therefore, there are no contradictions. Note that this naive emergence of the extra dimension is only valid for the low energy region. The Hilbert space of the CFT contains much more states which will be the black hole states. These modes dominates in the counting of the number of the states and give the area law of the entropy instead of the volume law in the low energy region. Thus, the holographic principle is consistent with this naive emergence of the extra dimension.

\footnotetext{
${ }^{16}$ This identification for one particle states was given in [18]

${ }^{17}$ This identification of Hilbert spaces are also explicitly shown as follows. It is clear that the basis of the CFT states (3.9) can be taken as $\tilde{R}\left(\hat{b}_{n l m}^{\dagger}\right)|0\rangle$, where $\tilde{R}$ is a monomial and $\hat{b}_{n l m}^{\dagger}=$ $c_{n l} s_{(l, m)}^{\mu_{1} \mu_{2} \ldots \mu_{l}} \hat{P}_{\mu_{1}} \hat{P}_{\mu_{2}} \cdots \hat{P}_{\mu_{l}}\left(\hat{P}^{2}\right)^{n} \hat{\mathcal{O}}_{\Delta}$. Then, the map from the states in the Fock space $\tilde{R}\left(\hat{a}_{n l m}^{\dagger}\right)|0\rangle$ to these states can be upper-triangular matrix by ordering the basis with an appropriate alphabetical ordering such that $\hat{\mathcal{O}}_{\Delta}>\hat{P}_{1}>\hat{P}_{2}>\cdots$.

${ }^{18}$ Of course, the AdS/CFT and these theories are different. Most crucial difference might be the UV theory. For these theories, the UV limit are weak coupling theory, however, the AdS/CFT case it is strong coupling.
} 


\subsection{Construction of bulk local field}

In this subsection, we will construct an operator in $\mathrm{CFT}_{d}$ corresponding to the local operator in $\mathrm{AdS}_{d+1}$ using the equivalence of the states which was shown explicitly.

First, we will fix the normalization constant $c_{n l}$ of the state, such that $\left[\hat{a}_{n l m}, \hat{a}_{n^{\prime} l^{\prime} m^{\prime}}^{\dagger}\right]=$ $\delta_{n, n^{\prime}} \delta_{l, l^{\prime}} \delta_{m, m^{\prime}}$ which is equivalent to $\left\langle 0\left|\hat{a}_{n^{\prime} l^{\prime} m^{\prime}} \hat{a}_{n l m}^{\dagger}\right| 0\right\rangle=\delta_{n, n^{\prime}} \delta_{l, l^{\prime}} \delta_{m, m^{\prime}}$. Thus, we will compute the norm of

$$
c_{n l} s_{(l, m)}^{\mu_{1} \mu_{2} \ldots \mu_{l}} P_{\mu_{1}} P_{\mu_{2}} \cdots P_{\mu_{l}}\left(P^{2}\right)^{n}\left|\hat{\mathcal{O}}_{\Delta}\right\rangle,
$$

where we normalized the primary state such that $\left\langle 0\left|\hat{\mathcal{O}}_{\Delta}^{\dagger} \hat{\mathcal{O}}_{\Delta}\right| 0\right\rangle=1$ and $s_{(l, m)}^{\mu_{1} \mu_{2} \ldots \mu_{l}}$ is the coefficients in the polynomial representation of the spherical harmonics $Y_{l m}(\Omega)$ explained in appendix B. The two point correlation function of the primary field with scaling dimension $\Delta$ is determined by the symmetry, see for example [28], as

$$
\left\langle\mathcal{O}_{\Delta}(y) \mathcal{O}_{\Delta}(x)\right\rangle=\frac{1}{|x-y|^{2 \Delta}}=\left(y^{2}\right)^{-\Delta}\left\langle\Delta\left|e^{\tilde{y} \cdot \hat{K}} e^{\tilde{x} \cdot \hat{P}}\right| \Delta\right\rangle,
$$

where $\tilde{y}^{\mu}=y^{\mu} / y^{2}$. From this, we find

$$
\begin{aligned}
\mid s_{(l, m)}^{\mu_{1} \mu_{2} \ldots \mu_{l}} P_{\mu_{1}} & \left.P_{\mu_{2}} \cdots P_{\mu_{l}}\left(P^{2}\right)^{n}\left|\hat{\mathcal{O}}_{\Delta}\right\rangle\right|^{2} \\
= & \lim _{x, \tilde{y} \rightarrow 0} s_{\mu_{1} \mu_{2} \ldots \mu_{l}}^{(l, m)} \partial_{x}^{\mu_{1}} \partial_{x}^{\mu_{2}} \cdots \partial_{x}^{\mu_{l}}\left(\partial_{x}^{2}\right)^{n} s_{\nu_{1} \nu_{2} \ldots \nu_{l}}^{(l, m)} \partial_{\tilde{y}}^{\nu_{1}} \partial_{\tilde{y}}^{\nu_{2}} \cdots \partial_{\tilde{y}}^{\nu_{l}}\left(\partial_{\tilde{y}}^{2}\right)^{n} \\
& \times\left(\left(y^{2}\right)^{\Delta} \frac{1}{|x-y|^{2 \Delta}}\right) \\
= & \lim _{\tilde{y} \rightarrow 0} s_{\nu_{1} \nu_{2} \ldots \nu_{l}}^{(l, m)} \partial_{\tilde{y}}^{\nu_{1}} \partial_{\tilde{y}}^{\nu_{2}} \cdots \partial_{\tilde{y}}^{\nu_{l}} \\
& \times\left(\partial_{\tilde{y}}^{2}\right)^{n}\left(2^{2 n+l} \frac{\Gamma(\Delta+n+l)}{\Gamma(\Delta)} \frac{\Gamma\left(\Delta+1-\frac{d}{2}+n\right)}{\Gamma\left(\Delta+1-\frac{d}{2}\right)} \tilde{y}^{2 n} s_{\mu_{1} \mu_{2} \ldots \mu_{l}}^{(l, m)} \tilde{y}^{\mu_{1}} \tilde{y}^{\mu_{2}} \cdots \tilde{y}^{\mu_{l}}\right)
\end{aligned}
$$

where, in the final line, we have used the following two relations,

$$
\begin{aligned}
\left(\partial_{x}^{2}\right)^{n} \frac{1}{|x-y|^{2 \Delta}}= & \frac{1}{|x-y|^{2(\Delta+n)}} 2^{2 n} \frac{\Gamma(\Delta+n)}{\Gamma(\Delta)} \frac{\Gamma\left(\Delta+1-\frac{d}{2}+n\right)}{\Gamma\left(\Delta+1-\frac{d}{2}\right)} \\
\partial_{x}^{\mu_{1}} \partial_{x}^{\mu_{2}} \cdots \partial_{x}^{\mu_{l}} \frac{1}{|x-y|^{2(\Delta+n)}}= & \frac{1}{|x-y|^{2(\Delta+n+l)}} \\
& \times(-2)^{l}(x-y)^{\mu_{1}}(x-y)^{\mu_{2}} \cdots(x-y)^{\mu_{l}} \frac{\Gamma(\Delta+n+l)}{\Gamma(\Delta+n)},
\end{aligned}
$$

where $\mu_{a}$ is understood to be contracted by the symmetric traceless tensor $s_{\mu_{1} \mu_{2} \ldots \mu_{l}}^{(l, m)}$. Then, repeatedly using

$$
\left(\partial_{y}^{2}\right)\left(\left(y^{2}\right)^{n}\left(s_{(l, m)}^{\mu_{1} \mu_{2} \ldots \mu_{l}} y^{\mu_{1}} y^{\mu_{2}} \cdots y^{\mu_{l}}\right)\right)=4 n(n+l+d / 2-1)\left(y^{2}\right)^{n-1}\left(s_{(l, m)}^{\mu_{1} \mu_{2} \ldots \mu_{l}} y^{\mu_{1}} y^{\mu_{2}} \cdots y^{\mu_{l}}\right),
$$


we obtain the normalization constant as

$$
\begin{aligned}
\left|c_{n l}\right|^{-2}= & \left.\left|s_{(l, m)}^{\mu_{1} \mu_{2} \ldots \mu_{l}} P_{\mu_{1}} P_{\mu_{2}} \cdots P_{\mu_{l}}\left(P^{2}\right)^{n}\right| \hat{\mathcal{O}}_{\Delta}\right\rangle\left.\right|^{2} \\
= & 2^{4 n+l} \frac{\Gamma(\Delta+n+l)}{\Gamma(\Delta)} \frac{\Gamma\left(\Delta+1-\frac{d}{2}+n\right)}{\Gamma\left(\Delta+1-\frac{d}{2}\right)} n ! \frac{\Gamma\left(n+\frac{d}{2}+l\right)}{\Gamma\left(l+\frac{d}{2}\right)} \\
\quad & \quad s_{\nu_{1} \nu_{2} \ldots \nu_{l}}^{(l, m)} \partial_{\tilde{y}}^{\nu_{1}} \partial_{\tilde{y}}^{\nu_{2}} \cdots \partial_{\tilde{y}}^{\nu_{l}}\left(s_{\mu_{1} \mu_{2} \ldots \mu_{l}}^{(l, m)} \tilde{y}^{\mu_{1}} \tilde{y}^{\mu_{2}} \cdots \tilde{y}^{\mu_{l}}\right) \\
= & 2^{4 n+2 l} \frac{\Gamma(\Delta+n+l)}{\Gamma(\Delta)} \frac{\Gamma\left(\Delta+1-\frac{d}{2}+n\right)}{\Gamma\left(\Delta+1-\frac{d}{2}\right)} n ! \frac{\Gamma\left(n+\frac{d}{2}+l\right)}{\Gamma\left(\frac{d}{2}\right)} .
\end{aligned}
$$

As we will see later, this choice is consistent with the GKPW relation. In particular, for $l=0$, this becomes

$$
\begin{aligned}
\frac{1}{\left(c_{n 0}\right)^{2}} & \left.=\left|\left(P^{2}\right)^{n}\right| \hat{\mathcal{O}}_{\Delta}\right\rangle\left.\right|^{2} \\
& =2^{4 n} n ! \frac{\Gamma(\Delta+n)}{\Gamma(\Delta)} \frac{\Gamma\left(\Delta+1-\frac{d}{2}+n\right)}{\Gamma\left(\Delta+1-\frac{d}{2}\right)} \frac{\Gamma(n+d / 2)}{\Gamma(d / 2)} .
\end{aligned}
$$

We will take the phase of $c_{n l}$ such that

$$
c_{n l}=\left|c_{n l}\right|
$$

Before constructing the bulk local field, we will construct a simple bulk local state. In the bulk $\operatorname{AdS}_{d+1}$ description, a localized state for one particle at $t=0$ and $\rho=\rho_{0}$ is

$$
\hat{\phi}\left(t=0, \rho=\rho_{0}, \Omega\right)|0\rangle=\sum_{n, l, m} \psi_{n l m}\left(\rho=\rho_{0}\right) Y_{l m}(\Omega) \hat{a}_{n l m}^{\dagger}|0\rangle
$$

which is localized on $S^{d-1}$ except for $\rho_{0}=0 .{ }^{19}$ Note that we take the Heisenberg picture here. Thus we need to specify the time in order to give a physical meaning to the state. Indeed the localized state at $t=0$ is not localized at $t \neq 2 \pi \mathbf{Z}$. Here, we will concentrate on the state with $l=0$ and $\rho_{0}=0$ :

$$
\int d \Omega \hat{\phi}\left(t=0, \rho=\rho_{0}, \Omega\right)|0\rangle=\sum_{n=0}^{\infty} \psi_{n 00}(\rho=0) \hat{a}_{n 00}^{\dagger}|0\rangle=\sum_{n=0}^{\infty} \frac{1}{N_{n 0}} \hat{a}_{n 00}^{\dagger}|0\rangle,
$$

which is the state localized at a point $t=0, \rho=0$. We will consider general cases in the next subsection. For the one particle state, the one to one map between the normalized states in the bulk description and in the CFT description is ${ }^{20}$

$$
\hat{a}_{n 00}^{\dagger}|0\rangle \leftrightarrow c_{n 0}\left(P^{2}\right)^{n}\left|\hat{\mathcal{O}}_{\Delta}\right\rangle
$$

\footnotetext{
${ }^{19}$ A strictly localized state can not be normalized. Thus, in order to get a normalized (almost) localized state, we need to smear it.

${ }^{20}$ The phase can not be fixed in the large $N$ limit because if the Hilbert space and the Hamiltonian are given, then the quantum theory is fixed. Here, we require that the action of $\hat{P}^{2}$ is realized by the corresponding isometry of $\mathrm{AdS}_{d+1}$.
} 
Thus, the localized state is given in the CFT description ${ }^{21}$ as

$$
\sum_{n=0}^{\infty} \frac{1}{N_{n 0}} c_{n}\left(P^{2}\right)^{n}\left|\hat{\mathcal{O}}_{\Delta}\right\rangle=\sqrt{\frac{\Gamma(\Delta) \Gamma\left(\Delta+1-\frac{d}{2}\right)}{\Gamma(d / 2)}} \sum_{n=0}^{\infty} \frac{(-1)^{n} 2^{-2 n}}{n ! \Gamma(\Delta+1-d / 2+n)}\left(P^{2}\right)^{n}\left|\hat{\mathcal{O}}_{\Delta}\right\rangle
$$

which coincides with the twisted Ishibashi state [32] in [8, 10] and [9] except an extrra overall constant factor $\sqrt{\frac{\Gamma(\Delta)}{\Gamma\left(\Delta+1-\frac{d}{2}\right) \Gamma(d / 2)}}$. Note that the overall constant is irrelevant because we have not normalized the state.

Below, we will construct the bulk local operator by the identification of the states. We have seen that the one to one map between the operator in the bulk description and the one in the CFT description is given by

$$
\hat{a}_{n l m}^{\dagger} \longleftrightarrow c_{n l} s_{(l, m)}^{\mu_{1} \mu_{2} \ldots \mu_{l}} P_{\mu_{1}} P_{\mu_{2}} \cdots P_{\mu_{l}}\left(P^{2}\right)^{n} \hat{\mathcal{O}}_{\Delta}
$$

Here, we will decompose the local operator in the bulk description to positive and negative frequency modes as

$$
\hat{\phi}(t, \rho, \Omega)=\hat{\phi}^{+}(t, \rho, \Omega)+\hat{\phi}^{-}(t, \rho, \Omega)
$$

where $\hat{\phi}^{-}(t, \rho, \Omega)=\left(\hat{\phi}^{+}(t, \rho, \Omega)\right)^{\dagger}$. Thus, the local operator in the bulk description is represented by

$$
\begin{aligned}
\hat{\phi}^{+}(t=0, \rho, \Omega) & =\sum_{n, l, m} \psi_{n l m}(\rho) Y_{l m}(\Omega) \hat{a}_{n l m}^{\dagger} \\
& =\sum_{n, l, m} \psi_{n l m}(\rho) Y_{l m}(\Omega) c_{n l} s_{(l, m)}^{\mu_{1} \mu_{2} \ldots \mu_{l}} P_{\mu_{1}} P_{\mu_{2}} \cdots P_{\mu_{l}}\left(P^{2}\right)^{n} \hat{\mathcal{O}}_{\Delta} .
\end{aligned}
$$

Note that if we take $\rho \rightarrow 0$ limit, only $l=0$ modes remain:

$$
\psi_{n l m}(\rho)=\frac{1}{N_{n l}} \sin ^{l}(\rho) \cos ^{\Delta}(\rho){ }_{2} F_{1}\left(-n, \Delta+l+n, l+\frac{d}{2}, \sin ^{2}(\rho)\right) \rightarrow \frac{1}{N_{n 0}} .
$$

\subsection{Derivation of GKPW relation}

Now, let us consider the boundary value of the bulk operator (3.30), i.e. taking $\rho \rightarrow \pi / 2$ limit. Using

$$
{ }_{2} F_{1}\left(-n, \Delta+l+n, l+\frac{d}{2}, 1\right)=\frac{\Gamma(l+d / 2)}{\Gamma(n+l+d / 2)} \frac{\Gamma(d / 2-\Delta)}{\Gamma(d / 2-\Delta-n)},
$$

we obtain the following expression:

$$
\frac{c_{n l}}{N_{n l}}{ }_{2} F_{1}\left(-n, \Delta+l+n, l+\frac{d}{2}, 1\right)=\frac{2^{-2 n-l}}{n !} \frac{1}{\Gamma(n+l+d / 2)} \sqrt{\frac{\Gamma(d / 2) \Gamma(\Delta)}{\Gamma(\Delta+1-d / 2)}} .
$$

\footnotetext{
${ }^{21}$ This construction was done in [23] for $d=2$.
} 
With the hyper spherical Bessel function which is defined by

$$
\begin{aligned}
j_{l}^{d}(z) & \equiv z^{l} \sum_{n=0}^{\infty} \frac{(i z)^{2 n}}{(2 n) ! !(d+2 n+2 l-2) ! !} \\
& =\pi z^{l} \sum_{n=0}^{\infty} 2^{-2 n-l-d / 2} \frac{(i z)^{2 n}}{n ! \Gamma(n+l+d / 2)},
\end{aligned}
$$

the formula for the expansion of the plain wave in $\mathbf{R}^{d}$ by the spherical harmonics is given in [52]:

$$
\begin{aligned}
e^{i k_{\mu} x^{\mu}} & =(d-2) ! ! \sum_{l=0}^{\infty} i^{l} j_{l}^{d}(k r) \sum_{m} Y_{l m}^{*}\left(\Omega_{k}\right) Y_{l m}(\Omega) \\
& =\sum_{l=0}^{\infty} i^{l} \sqrt{\frac{\pi}{2}}(k r)^{l} \sum_{n=0}^{\infty} 2^{-2 n-l} \frac{\Gamma(d / 2)(i k r)^{2 n}}{n ! \Gamma(n+l+d / 2)} \sum_{m} Y_{l m}^{*}\left(\Omega_{k}\right) Y_{l m}(\Omega),
\end{aligned}
$$

where $r=\sqrt{x^{\mu} x_{\mu}}, k=\sqrt{k^{\mu} k_{\mu}}, \Omega$ and $\Omega_{k}$ are the angular variables for $x^{\mu}$ and $k^{\mu}$, respectively. Using this formula with $r=1$ and $k_{\mu}=-i P_{\mu}$, we obtain

$$
\lim _{\rho \rightarrow \pi / 2} \frac{\hat{\phi}^{+}(t=0, \rho, \Omega)}{\cos ^{\Delta}(\rho)}=\sqrt{\frac{\pi}{2}} \sqrt{\frac{\Gamma(\Delta)}{\Gamma(\Delta+1-d / 2) \Gamma(d / 2)}} e^{P_{\mu} x^{\mu}} \hat{\mathcal{O}}_{\Delta},
$$

where $x^{2}=1$, which means

$$
\lim _{\rho \rightarrow \pi / 2} \frac{\hat{\phi}^{+}(t=0, \rho, \Omega)}{\cos ^{\Delta}(\rho)}=\left.\sqrt{\frac{\pi}{2}} \sqrt{\frac{\Gamma(\Delta)}{\Gamma(\Delta+1-d / 2) \Gamma(d / 2)}} \hat{\mathcal{O}}_{\Delta}^{+}(x)\right|_{x^{2}=1} .
$$

The operator on the cylinder $\mathbf{R} \times S^{d-1}$ is given by $\mathcal{O}_{\Delta}^{c y}(\tau, \Omega)=\mathcal{O}_{\Delta}(x) e^{\Delta \tau}$ where $\tau=$ $\ln \left(x^{2}\right) / 2$ from the operator $\mathcal{O}_{\Delta}(x)$ which is radially quantized on $\mathbf{R}^{d}$. Using this operator, we find

$$
\lim _{\rho \rightarrow \pi / 2} \frac{\hat{\phi}(t, \rho, \Omega)}{\cos ^{\Delta}(\rho)}=\sqrt{\frac{\pi}{2}} \sqrt{\frac{\Gamma(\Delta)}{\Gamma(\Delta+1-d / 2) \Gamma(d / 2)}} \mathcal{O}_{\Delta}^{c y}(t, \Omega),
$$

where we have neglected the operators in $\mathcal{O}_{\Delta}^{c y}(t, \Omega)$ whose energies range from $-\Delta+1$ to $\Delta-1$ because of the large $N$ limit. ${ }^{22}$ Thus, the primary field in the $\mathrm{CFT}_{d}$ is given by the boundary value of the corresponding bulk operator with a constant factor. Such a relation was written in [5] and used as a starting point to construct the bulk local operator in [13].

The GKPW relation is essentially obtained from this relation. Indeed, schematically, with a background "non-normalizable" mode $\delta \phi=(\cos (\rho))^{\Delta^{-} \bar{\phi}}+\cdots$ with $\Delta^{-}=d-\Delta$, which is the solution of the e.o.m., induces

$$
\delta S=-\int_{\text {boundary }} d^{d} x\left((\cos (\rho))^{1-d} \delta \phi \frac{\partial}{\partial_{\rho}} \phi\right) \sim \int_{\text {boundary }} d^{d} x\left(\bar{\phi} \mathcal{O}_{\Delta}^{c y}\right),
$$

\footnotetext{
${ }^{22}$ These operators can be regarded as null states in the large $N$ limit because of the large $N$ factorization. However, for conserved currents, these includes the conserved charges which can not be neglected. This is not a contradiction because normalized conserved charges should appear in $\mathcal{O}_{\Delta}(t, \Omega)$ with $\mathcal{O}\left(N^{-\alpha}\right)$ factor with $\alpha>0$.
} 
which is the GKPW relation. ${ }^{23}$ Here, we have neglected the boundary term which corresponds to the renormalization. However, even including the boundary term, the relation (3.39) holds essentially as discussed in [34] in which the interactions in the bulk was also considered.

\subsection{Current}

Now we consider $\mathrm{CFT}_{d}$ with the symmetry current $J_{\nu}(x)$ which satisfies the conservation law $D^{\nu} J_{\nu}(x)=0$. We can define the corresponding operator as

$$
\lim _{x \rightarrow 0} J_{\nu}(x)|0\rangle=\hat{J}_{\nu}|0\rangle
$$

where the conservation law is represented by $P^{\nu} \hat{J}_{\nu} \equiv\left[\hat{P}^{\nu}, \hat{J}_{\nu}\right]=0$ and $\left[\hat{H}, \hat{J}_{\nu}\right]=(d-1) \hat{J}_{\nu}$ because the conformal dimension of the current is $d-1$. Furthermore, as for the scalar case, the "raising" operators can be defined by

$$
\hat{a}^{\dagger} \sim s^{\mu_{1} \mu_{2} \ldots \mu_{l^{\prime}}}\left(P^{2}\right)^{n} P_{\mu_{1}} P_{\mu_{2}} \cdots P_{\mu_{l^{\prime}}} \hat{J}_{\nu}
$$

which span the low energy states of $\mathrm{CFT}_{d}$ under the assumptions. Note that the with the rank $\left(l^{\prime}+1\right)$ tensor $s^{\mu_{1} \mu_{2} \ldots \mu_{l^{\prime}} \nu}$, is traceless for all indices and symmetric for the first $l^{\prime}$ indices. Thus, there are two different kind of the raising operators; symmetric and anti-symmetric for the indices $\mu$ and $\nu$. We will denote these as

$$
\hat{a}_{V}^{\dagger} \sim s_{V}^{\mu_{1} \mu_{2} \ldots \mu_{l+1}}\left(P^{2}\right)^{n} P_{\mu_{1}} P_{\mu_{2}} \cdots P_{\mu_{l}} \hat{J}_{\mu_{l+1}},
$$

where $s_{V}^{\mu_{1} \mu_{2} \ldots \mu_{l+1}}$ is symmetric for first $l$ indices and anti-symmetric for the last two indices, and

$$
\hat{a}_{S}^{\dagger} \sim s_{S}^{\mu_{1} \mu_{2} \ldots \mu_{l}}\left(P^{2}\right)^{n} P_{\mu_{1}} P_{\mu_{2}} \cdots P_{\mu_{l-1}} \hat{J}_{\mu_{l}}
$$

where $s_{S}^{\mu_{1} \mu_{2} \ldots \mu_{l}}$ is symmetric for all indices. Here, $l=1,2, \cdots$ and $n=0,1, \cdots$ for both of $\hat{a}_{V}^{\dagger}$ and $\hat{a}_{S}^{\dagger}$. The energy of $\hat{a}_{V}^{\dagger}$ and $\hat{a}_{S}^{\dagger}$ are $d-1+l+2 n$ and $d-1+l-1+2 n$, respectively, which are same as the raising operators for the free gauge field on $\operatorname{AdS}_{d+1}$. Therefore, the spectrum of the $\mathrm{CFT}_{d}$ with the current is identical to the gauge field on $\mathrm{AdS}_{d+1}$ under the assumptions of the sparse spectrum, the large $N$ factorization and the complete independence.

\subsection{Energy-momentum tensor}

Finally, we will consider energy momentum tensor $T_{\mu \nu}(x)$ which is a symmetric and traceless tensor and satisfies the conservation law $D^{\nu} T_{\mu \nu}(x)=0$. The analysis below is almost parallel with the one for the gauge field. We can define the corresponding operator as

$$
\lim _{x \rightarrow 0} T_{\mu \nu}(x)|0\rangle=\hat{T}_{\mu \nu}|0\rangle
$$

\footnotetext{
${ }^{23}$ Note that the GKPW relation is usually used for the theory on Eulidian $\mathrm{AdS}_{d+1}$ with $S^{d}$ boundary, which does not have a Hamiltonian formalism. For our case, the correlation function depends on the states and the boundary values. However, for example, if we consider the $\mathrm{CFT}_{d}$ on $S^{1} \times S^{d-1}$, the boundary values fix the partition function.
} 
This operator satisfies $P^{\nu} \hat{T}_{\mu \nu}=0$ and $\left[\hat{H}, \hat{T}_{\mu \nu}\right]=d \hat{T}_{\mu \nu}$. The "raising" operators can be defined by

$$
\hat{a}^{\dagger} \sim s^{\mu_{1} \mu_{2} \ldots \mu_{l^{\prime}} \nu_{1} \nu_{2}}\left(P^{2}\right)^{n} P_{\mu_{1}} P_{\mu_{2}} \cdots P_{\mu_{l^{\prime}}} \hat{T}_{\nu_{1} \nu_{2}},
$$

which span the states of $\mathrm{CFT}_{d}$. As for the gauge field, the constant tensor with $\left(l^{\prime}+2\right)$ indices, $s^{\mu_{1} \mu_{2} \ldots \mu_{l} \nu_{1} \nu_{2}}$, is traceless for all indices, symmetric for the first $l^{\prime}$ indices and symmetric for the last indices. Thus, this tensor is tensor product of the representations of $\mathrm{SO}(d)$ which are the Young diagram labeled by $\left[l^{\prime}, 0, \ldots, 0\right]$ and $[2,0, \ldots, 0]$. This is decomposed to the three irreducible representations, then there are three kinds of the raising operators. The first one is

$$
\hat{a}_{T}^{\dagger} \sim s_{T}^{\mu_{1} \mu_{2} \ldots \mu_{l+2}}\left(P^{2}\right)^{n} P_{\mu_{1}} P_{\mu_{2}} \cdots P_{\mu_{l}} \hat{T}_{\mu_{l+1} \mu_{l+2}}
$$

where $s_{T}^{\mu_{1} \mu_{2} \ldots \mu_{l+2}}$ is given by the anti-symmetrization of the pairs of the following indices: $\left(\mu_{1}, \mu_{l}+1\right)$ and $\left(\mu_{2}, \mu_{l}+2\right)$, and then the symmetrization for the first $l$ indices. This corresponds to the Young diagram labeled by $[l, 2,0, \ldots, 0]$. The second one is

$$
\hat{a}_{V}^{\dagger} \sim s_{V}^{\mu_{1} \mu_{2} \ldots \mu_{l+1}}\left(P^{2}\right)^{n} P_{\mu_{1}} P_{\mu_{2}} \cdots P_{\mu_{l-1}} \hat{T}_{\mu_{l} \mu_{l+1}},
$$

where $s_{V}^{\mu_{1} \mu_{2} \ldots \mu_{l+1}}$ is given by the anti-symmetrization of $\left(\mu_{l-1}, \mu_{l}+1\right)$ and $\left(\mu_{2}, \mu_{l}+2\right)$, and then the symmetrization for the first $l$ indices. This corresponds to the Young diagram labeled by $[l, 1,0, \ldots, 0]$. The final one is

$$
\hat{a}_{S}^{\dagger} \sim s_{S}^{\mu_{1} \mu_{2} \ldots \mu_{l}}\left(P^{2}\right)^{n} P_{\mu_{1}} P_{\mu_{2}} \cdots P_{\mu_{l-2}} \hat{T}_{\mu_{l-1} \mu_{l}},
$$

where $s_{S}^{\mu_{1} \mu_{2} \ldots \mu_{l}}$ is symmetric for all indices corresponds to the Young diagram labeled by $[l, 0,0, \ldots, 0]$. Here, $l=2,3, \cdots$ and $n=0,1, \cdots$ for all klinds of the raising operators. The energy of $\hat{a}_{T}^{\dagger}, \hat{a}_{V}^{\dagger}$ and $\hat{a}_{S}^{\dagger}$ are $d+l+2 n, d+l-1+2 n$ and $d+l-2+2 n$, respectively, which are same as the raising operators for the gravitational perturbations on $\operatorname{AdS}_{d+1}$. Therefore, the spectrum of the $\mathrm{CFT}_{d}$ for the energy momentum tensor is identical to the gravitational perturbations on $\mathrm{AdS}_{d+1}$ with the assumptions of the sparse spectrum, the large $N$ factorization and the complete independence.

We can construct the localized states and local field for the current and the energymomentum tensor as for the scalar field case. In order to do it explicitly, we need to compute the normalization constants of the states in $\mathrm{CFT}_{d}$. In this paper we will only consider some simple states. We hope to do for general cases in future. The two point function for the spin- $l$ traceless symmetric tensors are known to be given by

$$
\left\langle J^{\mu_{1} \cdots \mu_{l}}(x) J^{\nu_{1} \cdots \nu_{l}}(0)\right\rangle=C\left(\frac{I_{\nu_{1}}^{\left(\mu_{1}\right.}(x) \cdots I_{\nu_{l}}^{\left.\mu_{l}\right)}(x)}{x^{2 \Delta}}-\text { traces }\right),
$$

where $I_{\nu}^{\mu}(x)=\delta^{\mu}{ }_{\nu}-2 \frac{x^{\mu} x_{\nu}}{x^{2}}$ and $C$ is a constant. Now let us concentrate on the $\left(\hat{P}^{2}\right)^{n}\left|J^{\nu_{1} \cdots \nu_{l}}\right\rangle$ and compute the norm of this state. As in the scalar case, we can find

$$
\left.\sum_{\mu_{1}, \cdots, \mu_{l}}\left|\left(\hat{P}^{2}\right)^{n}\right| J^{\nu_{1} \cdots \nu_{l}}\right\rangle\left.\right|^{2}=\lim _{x, \tilde{y} \rightarrow 0}\left(\partial_{x}^{2}\right)^{n}\left(\partial_{y}^{2}\right)^{n}\left(\frac{C^{\prime} y^{2}}{(x-y)^{2}}\right)^{\Delta},
$$

where $C^{\prime}$ is a constant because the $\mu$ indices are contracted. This form is $l$ independent, thus the form of the localized state is same as the one for the scalar. 


\section{$4 \mathrm{AdS}_{3} / \mathrm{CFT}_{2}$}

For $\operatorname{AdS}_{3}(d=2)$ case, the fluctuations of the metric are not same as $d>2$ case. In this case, there are no bulk propagating degrees of freedom because the e.o.m. implies the geometry is locally $\mathrm{AdS}_{3}$. Indeed, we can check this fact by solving the e.o.m. with a gauge fixing condition of the diffeomorphism.

However, there is the famous subtlety for the gravitons on $\mathrm{AdS}_{3}$. A" gauge" transformation which changes the asymptotic behavior, ${ }^{24}$ is not regarded as a gauge transformation, i.e. it is not an identification of the configurations. It is often called a large gauge transformation. If the transformation keeps the boundary condition we imposed, then, such large gauge transformations, which is called the asymptotic symmetry, are physically acceptable and configurations generated by those should be regarded as excited states. With the boundary conditions on the asymptotically $\mathrm{AdS}_{3}$ metric given in [35], the asymptotic symmetry is the Virasoro algebra which generates the states from the vacuum which are called the boundary gravitons. Thus it is expected that the semi-classical quantization around the vacuum gives the states which are Verma module of the Virasoro algebra for the identity operator without null vectors. This was claimed in [36] and was explicitly shown by computing the one-loop partition function using the heat kernel [37] and also using the SUSY localization $[38-40] .{ }^{25}$

It is obvious that these states can not be localized in the bulk by taking a linear combinations. We can construct the (twisted) Ishibashi state of the $\mathrm{SO}(2,2)$ by acting $\hat{P}^{2} \sim L_{-1} \tilde{L}_{-1}$ to the primary state $\left(L_{-2}\right)\left(\tilde{L}_{-2}\right)|0\rangle$, which satisfies the condition given in [9]. ${ }^{26}$ However, this condition is only a necessary condition for a localized state.

Note that the states which are given by acting $L_{-n}, \tilde{L}_{-n}$ with $n>1$ to the vacuum correspond to the states with the boundary gravitons which spread over the bulk and are not localized on the boundary because the configurations for them depend non-trivially on the radial coordinate. Thus, such states, which include cross cap states of the Virasoro algebra, ${ }^{27}$ can not be localized.

For a gauge field in $\mathrm{AdS}_{3}$, we can apply the discussions on the previous sections and the states in $\mathrm{CFT}_{2}$ generated by the corresponding current reproduce the fluctuations of the gauge field in $\mathrm{AdS}_{3}$. However, in the discussion, we assumed complete independence of the states except the current conservation. Usually, the current in $\mathrm{CFT}_{2}$ is factorized to the holomorphic and the anti-holomorphic parts which means that the relation $d J=0$ holds adding to the conservation law $d * J=0$. This violates the complete independence and then such holomorphic current can not correspond to the bulk propagating gauge field. It is expected that this holomorphic current corresponds to the three dimensional Chern-Simons theory in $\mathrm{AdS}_{3}$ because there are propagating degree of freedoms are only near the boundary, like the Virasoro algebra case. Indeed,with an appropriate boundary condition, Chern-Simons theory on the manifold with the boundary was show

\footnotetext{
${ }^{24}$ Here, the asymptotic behavior is said to be changed if the energy of the configuration is changed.

${ }^{25}$ For the higher spin theories, these results have been extended in [41, 42].

${ }^{26}$ From $\left(L_{-2}\right)|0\rangle$ or $\left(\tilde{L}_{-2}\right)|0\rangle$, we can not construct the twisted Ishibashi state because $\hat{P}^{2}\left(L_{-2}\right)|0\rangle=$ $\hat{P}^{2}\left(\tilde{L}_{-2}\right)|0\rangle=0$.

${ }^{27}$ In [33], the relation between the Virasoro symmetry and local states was discussed.
} 
to be described by the Wess-Zumino-Wiiten model which has the holomorphic and anitholomorphic currents [43].

\section{Thermal states}

We have considered the fluctuations around the vacuum, i.e. $\mathrm{AdS}_{d+1}$. To extend this background to the black hole in asymptotic $\mathrm{AdS}_{d+1}$ is interesting, however, there are problems for finding the normalized eigen modes in such a background because the black hole solution is not static and there are dissipations into the black hole.

However, in the dual $\mathrm{CFT}_{d}$ side, there may be typical states for a thermal equilibrium state, which is in a sense static. This finite temperature state is expected to correspond the black hole and the thermal gas around it. The Hawking radiations and the thermal gas are in equilibrium. For a very large black hole, we expect to rely the semi-classical picture outside a stretched horizon which is close to the horizon and impose, for example, the Dirichlet boundary condition at the stretched horizon in order to understand a qualitative property of the system, This hypothetical boundary is called a brick wall and was introduced by 't Hooft [44]. With this boundary, the system is approximately static and we can count the number of the low energy modes approximately [45]. ${ }^{28}$ The number is divergent by moving the location of the boundary to the horizon because of the warp factor near the horizon. This divergence may be physical and corresponds to a deconfinement phenomena in the dual gauge theory because in the deconfinement phase the number of low energy fields will be proportional to $N^{2}$ which is divergent in the large $N$ limit [45]. Here, we think the number of low energy fields can be defined as an approximate notion. This suggest that the degrees of freedom of the dual gauge theory only construct the space outside the (stretched) horizon for a very old and large black hole and there is the brick wall (or a fire wall [48]). This picture is consistent with the fuzzball conjecture [49-51].

\section{Acknowledgments}

S.T. would like to thank Shigeki Sugimoto, Masaki Shigemori, Tadashi Takayangi and Shuichi Yokoyama for useful discussions. This work was supported by JSPS KAKENHI Grant Number 17K05414.

\footnotetext{
${ }^{28}$ In [44], the divergence of the partition function was discussed. This divergence can be renormalized by the redefinition of the Newton constant $[46,47]$. Here, we focus on the number of the low energy modes, instead of the divergence of the partition function although this divergence comes from the infinitely many low energy modes.
} 


\section{A Symmetric tensor harmonics on $S^{d-1}$}

The rank $r$ symmetric (traceless) tensor harmonics on unit radius $S^{d-1}, Y_{i_{1}, i_{2}, \cdots, i_{r}}^{l m}\left(z^{i}\right)$, is defined such that $Y_{i_{1}, i_{2}, \cdots, i_{r}}^{l m}$ is totally symmetric for the indices $i_{k}$ and

$$
\begin{aligned}
D^{i} D_{i} Y_{i_{1}, i_{2}, \cdots, i_{r}}^{l m} & =(-l(l+d-2)+r) Y_{i_{1}, i_{2}, \cdots, i_{r}}^{l m}, \\
D^{i} Y_{i, i_{2}, \cdots, i_{r}}^{l m} & =0 \\
g_{S^{d-1}}^{i j} Y_{i, j, i_{3} \cdots, i_{r}}^{l m} & =0
\end{aligned}
$$

where $z^{i}(i=1,2, \cdots, d-1)$ is the coordinate $D_{i}$ is the covariant derivative, and $g_{S^{d-1}}^{i j}$ is the inverse metric of unit radius $S^{d-1}$. Here, $l=r, r+1, r+2, \cdots$ and $m$ runs from 1 to the number of the independent harmonics which depends on $l$. This harmonics $Y_{i_{1}, i_{2}, \cdots, i_{r}}^{(r) l m}$ is the unitary representation of $\mathrm{SO}(d)$ which corresponds to the Young diagram labeled by $[l, r, 0, \ldots, 0]$. More details for the symmetric tensor harmonics, see [53-56].

It is known [25] that the vector field $v_{i}\left(z^{i}\right)$ on $S^{d-1}$ can be uniquely decomposed to

$$
v_{i}\left(z^{i}\right)=\sum_{l=1, m} v_{l m}^{V} Y_{i}^{l m}\left(z^{i}\right)+\sum_{l=1, m} v_{l m}^{S} D_{i} Y^{l m}\left(z^{i}\right)
$$

where $v_{l m}^{V}$ and $v_{l m}^{S}$ are constant. For the second rank symmetric tensor field $t_{i j}\left(z^{i}\right)$, the following unique decomposition is possible:

$$
\begin{aligned}
t_{i j}\left(z^{i}\right)= & \sum_{l=2, m} t_{l m}^{T} Y_{j i}^{l m}\left(z^{i}\right)+\sum_{l=2, m} t_{l m}^{V}\left(D_{i} Y_{j}^{l m}\left(z^{i}\right)+D_{j} Y_{i}^{l m}\left(z^{i}\right)\right) \\
& +\sum_{l=2, m} t_{l m}^{S}\left(D_{i} D_{j}-\frac{1}{d-1} g_{i j}^{S^{d-1}} D_{k} D_{k}\right) Y^{l m}\left(z^{i}\right)+\sum_{l=0, m} t_{l m}^{\text {trace }} \frac{1}{d-1} g_{i j}^{S^{d-1}} Y^{l m}\left(z^{i}\right) .
\end{aligned}
$$

\section{B Normalization of spherical harmonics}

The spherical harmonics on $S^{d-1}$ is obtained by the harmonic polynomials on $\mathbf{R}^{d}$. Here, the harmonic polynomial of order $l$ is a homogeneous polynomial of order $l$ such that

$$
\sum_{\mu=1}^{d} \frac{\partial}{\partial x^{\mu}} \frac{\partial}{\partial x^{\mu}} h_{l}(x)=0
$$

where $x^{\mu}$ is the coordinate on $\mathbf{R}^{d}$. Then, we define

$$
Y_{l m}(\Omega) \equiv r^{-l} h_{l}(x),
$$

where $r=\sqrt{\sum_{i=\mu}^{d}\left(x_{\mu}\right)^{2}}$. This function depends only the angular variables on $\mathbf{R}^{d}$, which was denoted as $\Omega$ and $m$ represents a label of a basis of the harmonic polynomials of order $l$. Of course, this $\Omega$ can be regarded as coordinates on $S^{d-1}$ and $Y_{l m}(\Omega)$ is a function on $S^{d-1}$. This satisfies

$$
\left(\Delta_{S^{d-1}}-l(l+d-2)\right) Y_{l m}(\Omega)=0,
$$


where $\Delta_{S^{d-1}}$ is the Laplacian on $S^{d-1}$, because

$$
\sum_{\mu=1}^{d} \frac{\partial}{\partial x^{\mu}} \frac{\partial}{\partial x^{\mu}}=\frac{1}{r^{d-1}} \frac{\partial}{\partial r} r^{d-1} \frac{\partial}{\partial r}-\frac{\Delta_{S^{d-1}}}{r^{2}} .
$$

Thus, $Y_{l m}(\Omega)$ is the spherical harmonics on $S^{d-1}$ and we can show that any spherical harmonics can be obtained by this.

We will denote the spherical harmonics as

$$
Y_{l m}(\Omega)=r^{-l} s_{(l, m)}^{\mu_{1} \mu_{2} \ldots \mu_{l}} x_{\mu_{1}} x_{\mu_{2}} \cdots x_{\mu_{l}},
$$

which are normalized such that

$$
\frac{1}{\int d \Omega} \int d \Omega Y_{l m}(\Omega) Y_{l^{\prime} m^{\prime}}(\Omega)=\delta_{l l^{\prime}} \delta_{m m^{\prime}}
$$

where $\prod_{\mu=1}^{d} d x^{\mu}=r^{d-1} d r d \Omega$ and $\int d \Omega=\frac{2(\pi)^{d / 2}}{\Gamma(d / 2)}$. Here, $s_{(l, m)}^{\mu_{1} \mu_{2} \ldots \mu_{l}}$ is a symmetric traceless tensor because of (B.1). For a monomial, we can compute the integration over the angular variable $\Omega$ as

$$
\begin{aligned}
\int d \Omega r^{-l} \prod_{\mu=1}^{d}\left(x^{\mu}\right)^{n_{\mu}} & =\frac{\left(\int_{0}^{\infty} d r r^{d-1+l} e^{-r^{2}}\right)\left(\int d \Omega r^{-l} \prod_{\mu=1}^{d}\left(x^{\mu}\right)^{n_{\mu}}\right)}{\int_{0}^{\infty} d r r^{d-1+l} e^{-r^{2}}} \\
& =\frac{2 \int_{-\infty}^{\infty} d^{d} x \prod_{\mu=1}^{d}\left(x^{\mu}\right)^{n_{\mu}} e^{-\sum_{\nu=1}^{d}\left(x^{\nu}\right)^{2}}}{\Gamma\left(\frac{d+l}{2}\right)},
\end{aligned}
$$

where $l=\sum_{\mu=1}^{d} n_{\mu}$ and $n_{\mu}$ is a non negative integer. Then, from $\int d \Omega=\int d \Omega Y_{l m}(\Omega)^{2}$, we find the following constraint on $s_{(l, m)}^{\mu_{1} \mu_{2} \ldots \mu_{l}}$ :

$$
\begin{aligned}
\Gamma(l+ & d / 2) \delta_{l l^{\prime}} \delta_{m m^{\prime}} \int d \Omega \\
& =2 \int_{-\infty}^{\infty}\left(\prod_{\mu=1}^{d} d x^{\mu}\right) e^{-\sum_{\mu=1}^{d}\left(x^{\mu}\right)^{2}}\left(s_{\left(l^{\prime}, m^{\prime}\right)}^{\nu_{1} \nu_{2} \ldots \nu_{l^{\prime}}} x_{\nu_{1}} x_{\nu_{2}} \cdots x_{\nu_{l^{\prime}}}\right)\left(s_{(l, m)}^{\mu_{1} \mu_{2} \ldots \mu_{l}} x_{\mu_{1}} x_{\mu_{2}} \cdots x_{\mu_{l}}\right) .
\end{aligned}
$$

Note that the r.h.s. of this equation is represented as a correlation function of zerodimensional free fields theory and

$$
0=\int d \Omega Y_{l m}(\Omega) \sim \int_{-\infty}^{\infty}\left(\prod_{\mu=1}^{d} d x^{\mu}\right) e^{-\sum_{\mu=1}^{d}\left(x^{\mu}\right)^{2}} s_{(l, m)}^{\mu_{1} \mu_{2} \ldots \mu_{l}} x_{\mu_{1}} x_{\mu_{2}} \cdots x_{\mu_{l}},
$$

for $l \neq 0$. This means that there are no self-contractions, thus we can rewrite the constraint as

$$
2^{l} \frac{\Gamma(l+d / 2)}{\Gamma(d / 2)} \delta_{l l^{\prime}} \delta_{m m^{\prime}}=\left(s_{\left(l^{\prime}, m^{\prime}\right)}^{\nu_{1} \nu_{2} \ldots \nu_{l^{\prime}}} \partial_{\nu_{1}} \partial_{\nu_{2}} \cdots \partial_{\nu_{l^{\prime}}}\right)\left(s_{(l, m)}^{\mu_{1} \mu_{2} \ldots \mu_{l}} x_{\mu_{1}} x_{\mu_{2}} \cdots x_{\mu_{l}}\right) .
$$

This expression will be used for the computation of the normalization of the CFT states. 
Open Access. This article is distributed under the terms of the Creative Commons Attribution License (CC-BY 4.0), which permits any use, distribution and reproduction in any medium, provided the original author(s) and source are credited.

\section{References}

[1] J.M. Maldacena, The large- $N$ limit of superconformal field theories and supergravity, Int. J. Theor. Phys. 38 (1999) 1113 [Adv. Theor. Math. Phys. 2 (1998) 231] [hep-th/9711200] [INSPIRE].

[2] S.S. Gubser, I.R. Klebanov and A.M. Polyakov, Gauge theory correlators from noncritical string theory, Phys. Lett. B 428 (1998) 105 [hep-th/9802109] [INSPIRE].

[3] E. Witten, Anti-de Sitter space and holography, Adv. Theor. Math. Phys. 2 (1998) 253 [hep-th/9802150] [INSPIRE].

[4] V. Balasubramanian, P. Kraus and A.E. Lawrence, Bulk versus boundary dynamics in Anti-de Sitter space-time, Phys. Rev. D 59 (1999) 046003 [hep-th/9805171] [INSPIRE].

[5] T. Banks, M.R. Douglas, G.T. Horowitz and E.J. Martinec, AdS dynamics from conformal field theory, hep-th/9808016 [INSPIRE].

[6] I. Heemskerk, J. Penedones, J. Polchinski and J. Sully, Holography from conformal field theory, JHEP 10 (2009) 079 [arXiv: 0907.0151] [INSPIRE].

[7] A.L. Fitzpatrick and J. Kaplan, AdS field theory from conformal field theory, JHEP 02 (2013) 054 [arXiv: 1208.0337] [INSPIRE].

[8] M. Miyaji et al., Continuous multiscale entanglement renormalization ansatz as holographic surface-state correspondence, Phys. Rev. Lett. 115 (2015) 171602 [arXiv:1506.01353] [INSPIRE].

[9] Y. Nakayama and H. Ooguri, Bulk locality and boundary creating operators, JHEP 10 (2015) 114 [arXiv: 1507.04130] [INSPIRE].

[10] H. Verlinde, Poking holes in AdS/CFT: bulk fields from boundary states, arXiv:1505.05069 [INSPIRE].

[11] I. Bena, On the construction of local fields in the bulk of $A d S_{5}$ and other spaces, Phys. Rev. D 62 (2000) 066007 [hep-th/9905186] [INSPIRE].

[12] A. Hamilton, D.N. Kabat, G. Lifschytz and D.A. Lowe, Local bulk operators in AdS/CFT: a boundary view of horizons and locality, Phys. Rev. D 73 (2006) 086003 [hep-th/0506118] [INSPIRE].

[13] A. Hamilton, D.N. Kabat, G. Lifschytz and D.A. Lowe, Holographic representation of local bulk operators, Phys. Rev. D 74 (2006) 066009 [hep-th/0606141] [INSPIRE].

[14] S. El-Showk and K. Papadodimas, Emergent spacetime and holographic CFTs, JHEP 10 (2012) 106 [arXiv:1101.4163] [INSPIRE].

[15] D. Kabat, G. Lifschytz and D.A. Lowe, Constructing local bulk observables in interacting AdS/CFT, Phys. Rev. D 83 (2011) 106009 [arXiv:1102.2910] [INSPIRE].

[16] D. Kabat, G. Lifschytz, S. Roy and D. Sarkar, Holographic representation of bulk fields with spin in AdS/CFT, Phys. Rev. D 86 (2012) 026004 [arXiv:1204.0126] [INSPIRE]. 
[17] D. Kabat and G. Lifschytz, CFT representation of interacting bulk gauge fields in AdS, Phys. Rev. D 87 (2013) 086004 [arXiv:1212.3788] [INSPIRE].

[18] A.L. Fitzpatrick, J. Kaplan and M.T. Walters, Universality of long-distance AdS physics from the CFT bootstrap, JHEP 08 (2014) 145 [arXiv:1403.6829] [INSPIRE].

[19] D. Kabat and G. Lifschytz, Bulk equations of motion from CFT correlators, JHEP 09 (2015) 059 [arXiv: 1505. 03755] [INSPIRE].

[20] D. Kabat and G. Lifschytz, Locality, bulk equations of motion and the conformal bootstrap, JHEP 10 (2016) 091 [arXiv:1603.06800] [INSPIRE].

[21] K. Goto, M. Miyaji and T. Takayanagi, Causal evolutions of bulk local excitations from CFT, JHEP 09 (2016) 130 [arXiv: 1605.02835] [INSPIRE].

[22] J.-W. Kim, Explicit reconstruction of the entanglement wedge, JHEP 01 (2017) 131 [arXiv: 1607.03605] [INSPIRE].

[23] K. Goto and T. Takayanagi, CFT descriptions of bulk local states in the AdS black holes, JHEP 10 (2017) 153 [arXiv: 1704.00053] [INSPIRE].

[24] P. Breitenlohner and D.Z. Freedman, Positive energy in Anti-de Sitter backgrounds and gauged extended supergravity, Phys. Lett. B 115 (1982) 197.

[25] A. Ishibashi and R.M. Wald, Dynamics in nonglobally hyperbolic static space-times. 3. Anti-de Sitter space-time, Class. Quant. Grav. 21 (2004) 2981 [hep-th/0402184] [INSPIRE].

[26] J.D. Qualls, Lectures on conformal field theory, arXiv:1511.04074 [INSPIRE].

[27] S. Rychkov, EPFL lectures on conformal field theory in $D \geq 3$ dimensions, arXiv: 1601.05000.

[28] D. Simmons-Duffin, The conformal bootstrap, arXiv:1602.07982 [INSPIRE].

[29] M. Duetsch and K.-H. Rehren, Generalized free fields and the AdS-CFT correspondence, Annales Henri Poincaré 4 (2003) 613 [math-ph/0209035] [INSPIRE].

[30] N. Arkani-Hamed, A.G. Cohen and H. Georgi, (De)constructing dimensions, Phys. Rev. Lett. 86 (2001) 4757 [hep-th/0104005] [INSPIRE].

[31] C.T. Hill, S. Pokorski and J. Wang, Gauge invariant effective Lagrangian for Kaluza-Klein modes, Phys. Rev. D 64 (2001) 105005 [hep-th/0104035] [INSPIRE].

[32] N. Ishibashi, The boundary and crosscap states in conformal field theories, Mod. Phys. Lett. A 4 (1989) 251 [INSPIRE].

[33] Y. Nakayama and H. Ooguri, Bulk local states and crosscaps in holographic CFT, JHEP 10 (2016) 085 [arXiv : 1605.00334] [INSPIRE].

[34] D. Harlow and D. Stanford, Operator dictionaries and wave functions in AdS/CFT and $d S / C F T$, arXiv:1104.2621 [INSPIRE].

[35] J.D. Brown and M. Henneaux, Central charges in the canonical realization of asymptotic symmetries: an example from three-dimensional gravity, Commun. Math. Phys. 104 (1986) 207 [INSPIRE].

[36] A. Maloney and E. Witten, Quantum gravity partition functions in three dimensions, JHEP 02 (2010) 029 [arXiv:0712.0155] [INSPIRE].

[37] S. Giombi, A. Maloney and X. Yin, One-loop partition functions of 3D gravity, JHEP 08 (2008) 007 [arXiv:0804.1773] [INSPIRE]. 
[38] N. Iizuka, A. Tanaka and S. Terashima, Exact path integral for 3D quantum gravity, Phys. Rev. Lett. 115 (2015) 161304 [arXiv:1504.05991] [INSPIRE].

[39] M. Honda, N. Iizuka, A. Tanaka and S. Terashima, Exact path integral for $3 D$ quantum gravity II, Phys. Rev. D 93 (2016) 064014 [arXiv:1510.02142] [InSPIRE].

[40] S. Sugishita and S. Terashima, Exact results in supersymmetric field theories on manifolds with boundaries, JHEP 11 (2013) 021 [arXiv: 1308.1973] [INSPIRE].

[41] M.R. Gaberdiel, R. Gopakumar and A. Saha, Quantum W-symmetry in AdS $S_{3}$, JHEP 02 (2011) 004 [arXiv: 1009.6087] [INSPIRE].

[42] M. Honda, N. Iizuka, A. Tanaka and S. Terashima, Exact path integral for 3D higher spin gravity, Phys. Rev. D 95 (2017) 046016 [arXiv:1511.07546] [INSPIRE].

[43] E. Witten, Quantum field theory and the Jones polynomial, Commun. Math. Phys. 121 (1989) 351 [INSPIRE].

[44] G. 't Hooft, On the quantum structure of a black hole, Nucl. Phys. B 256 (1985) 727 [INSPIRE].

[45] N. Iizuka and S. Terashima, Brick walls for black holes in AdS/CFT, Nucl. Phys. B 895 (2015) 1 [arXiv: 1307.5933] [INSPIRE].

[46] L. Susskind and J. Uglum, Black hole entropy in canonical quantum gravity and superstring theory, Phys. Rev. D 50 (1994) 2700 [hep-th/9401070] [INSPIRE].

[47] J.-G. Demers, R. Lafrance and R.C. Myers, Black hole entropy without brick walls, Phys. Rev. D 52 (1995) 2245 [gr-qc/9503003] [INSPIRE].

[48] A. Almheiri, D. Marolf, J. Polchinski and J. Sully, Black holes: complementarity or firewalls?, JHEP 02 (2013) 062 [arXiv: 1207.3123] [INSPIRE].

[49] S.D. Mathur, The fuzzball proposal for black holes: an elementary review, Fortsch. Phys. 53 (2005) 793 [hep-th/0502050] [INSPIRE].

[50] S.D. Mathur, The information paradox: a pedagogical introduction, Class. Quant. Grav. 26 (2009) 224001 [arXiv:0909.1038] [INSPIRE].

[51] S.D. Mathur, What the information paradox is not, arXiv:1108.0302 [INSPIRE].

[52] J. Avery, Hyperspherical Harmonics; applications in quantum theory, Kluwer Academic Publishers, Dordrecht The Netherlands (1989).

[53] A. Chodos and E. Myers, Gravitational contribution to the Casimir energy in Kaluza-Klein theories, Annals Phys. 156 (1984) 412 [InSPIRE].

[54] M.A. Rubin and C.R. Ordonez, Symmetric tensor eigen spectrum of the laplacian on $n$ spheres, J. Math. Phys. 26 (1985) 65 [INSPIRE].

[55] M.A. Rubin and C.R. Ordonez, Eigenvalues and degeneracies for n-dimensional tensor spherical harmonics, UTTG-10-83 (1983).

[56] A. Higuchi, Symmetric tensor spherical harmonics on the $N$ sphere and their application to the de Sitter group $\mathrm{SO}(N, 1)$, J. Math. Phys. 28 (1987) 1553 [Erratum ibid. 43 (2002) 6385] [INSPIRE]. 